\title{
Archives de sciences sociales des religions
}

122 | avril - juin 2003

Varia

Jacques Blamont, Le Lion et le moucheron. Histoire des Marranes de Toulouse

Paris, Odile Jacob, 2000, 464 p. (bibliogr., index, tablx.).

\section{Colette Zytnicki}

\section{(2) OpenEdition}

\section{Journals}

Édition électronique

URL : http://journals.openedition.org/assr/1191

DOI : $10.4000 /$ assr. 1191

ISSN : $1777-5825$

\section{Éditeur}

Éditions de l'EHESS

Édition imprimée

Date de publication : 1 avril 2003

Pagination : $59-157$

ISBN : 2-222-96732-5

ISSN : 0335-5985

\section{Référence électronique}

Colette Zytnicki, « Jacques Blamont, Le Lion et le moucheron. Histoire des Marranes de Toulouse»,

Archives de sciences sociales des religions [En ligne], 122 | avril - juin 2003, document 122.9, mis en

ligne le 10 novembre 2005, consulté le 24 septembre 2020. URL : http://journals.openedition.org/assr/ 1191 ; DOl : https://doi.org/10.4000/assr.1191 
On peut se demander si le tikkoun messianique, la réparation de la fin des temps, n'est pas une restauration, ce qui nous ramènerait à la conception cyclique du temps. Or, comme le montre l'A. - en s'appuyant sur les travaux de Gershom Scholem - le restauré est toujours plus riche que le commencement, le monde à venir est une invention et non un simple retour à l'origine. Il s'agit de la dimension foncièrement rétroprojective du messianisme juif, qui conjugue inséparablement la restauration et l'utopie.

Le même raisonnement vaut pour l'impératif juif de la remémoration (Zakhor) : dans le rituel du seder, la pâque juive, l'obligation de se souvenir du passé se fait injonction utopique de réinventer ici et maintenant l'épopée libératrice.

Dans un des passages les plus intéressants du livre l'A. propose deux types opposés de nostalgie : 1) la nostalgie passéiste des doctrines restauratrices, qui veut "transformer l'avenir en revenir" et échapper à l'irréversibilité du temps par une rétrogradation impossible. 2) la nostalgie rétroprojective, qui s'inspire de ce qui ne fut point et aurait pu être, des espoirs déçus du passé, pour penser l'avenir et nourrir l'espoir. Cette nostalgie "productive", qui trouve dans Rousseau sa figure moderne inaugurale, est aussi celle du messianisme juif.

Pour le messianisme, l'espérance est toujours traversée par le désespoir, elle consiste à espérer l'inespéré, à attendre contre toute attente. Elle est aux antipodes de l'attente confiante et optimiste des philosophies du progrès, dont la croyance aveugle dans la Zuversicht (Providence) historique est, selon Ernst Bloch, le contraire du Principe Espérance.

Plus discutable me semble la tentative de G.B. de trouver des convergences ou des "consonances", entre le messianisme juif et l'œuvre de philosophes comme Kant, Schelling, Nietzsche ou Heidegger. Par ailleurs, il me paraît sous-estimer la dimension eschatologique, la dimension catastrophique, "révolutionnaire", apocalyptique de la fracture messianique du temps, si fortement mise en évidence par Scholem et ici insuffisamment prise en compte.

Quelles que soient les réserves qu'on peut avoir sur telle ou telle question, ce livre n'est pas moins un apport précieux à l'intelligence philosophique de la singularité de la conception messianique juive du temps.

Michael Löwy.
Le Lion et le moucheron. Histoire des Marranes de Toulouse. Paris, Odile Jacob, 2000, 464 p. (bibliogr., index, tablx.).

Ce livre est un ouvrage de piété, non pas dans le sens strictement religieux du terme, mais en tant qu'hommage rendu par l'auteur à certains de ses ancêtres marranes, découverts par hasard au cours d'une enquête généalogique. En dénouant les fils du passé familial, J.B. a fait resurgir la figure d'un certain Roque de Léon condamné pour judaïsme en 1685 à Toulouse. À partir de cette figure et de sa famille, tout un univers s'est alors révélé à lui, celui de ces juifs chassés d'Espagne puis du Portugal, trouvant un havre fragile et provisoire en France, pour s'installer définitivement en terre protestante plus accueillante. L'A. a donc suivi la piste des Marranes qui, pendant un siècle, vont toujours fuyant, mais toujours travaillant, toujours négociant, chrétiens au dehors, juifs au dedans.

De cette histoire, il l'avoue, il aurait pu faire un roman historique. Le sujet dramatique à souhait, fertile en rebondissements, mais aussi troué de larges zones d'ombre, s'y prêtait bien. Mais c'est en historien qu'il a finalement décidé de l'aborder. Pour deux raisons. La première est qu'il lui a semblé nécessaire "d'exhumer » ce groupe doublement occulté : du temps de son vivant d'abord, obligé de se cacher derrière des masques qui n'impressionnaient personne; après sa mort ensuite. Car les Marranes de Toulouse ont laissé peu de traces dans l'histoire et ont peu attiré l'attention, à part celle de quelques érudits au nombre desquels il faut ranger E. Szapiro qui depuis trente ans s'est fait l'historien de ceux que J.B. appelle les «cloportes». Image forte, choquante, qui doit à la fois à Kafka et à la posture de l'historien obligé de retourner les pierres entendons les archives - pour trouver, bien cachés depuis des siècles, ces oubliés de l'histoire.

La seconde raison qui l'a poussé à faire œuvre d'historien tient à sa propre situation. Physicien renommé qui a présidé aux destinées du CNES (Centre national d'études spatiales), en homme qui «a consacré (sa) vie à porter (son) pays au premier plan », il a été amené à s'interroger sur les causes d'une certaine défaite de la France face à la puissance montante anglaise, défaite qui selon lui prend ses racines dans la seconde moitié du XVII ${ }^{\mathrm{e}}$ siècle. Tandis que l'Angleterre comprenait que la prospérité se construisait dans les échanges et sur les mers, les Français faisaient le choix de se replier sur leurs terres, entendons ceci au 
sens économique et géopolitique du terme. Dans ce questionnement, les Marranes jouent un rôle clé. Par leur activité, leurs réseaux, ils représentaient l'ouverture au monde et à la nouvelle économie émergente : la France de Louis XIV les a chassés, par fanatisme, mais aussi parce qu'elle n'avait pas su repérer en eux les ferments de cette richesse qu'ils vont apporter ailleurs, en Hollande et en Grande-Bretagne.

C'est dire si le propos de J.B. est ambitieux. Car il se place dans la double perspective de contribuer à l'histoire d'un groupe oublié et d'apporter des éléments à l'explication du développement général de notre pays. Y réussit-il ? Concernant le premier objectif, sans nul doute. Grâce à un patient travail d'archives - l'A. a dû dépouiller nombre d'archives notariales, fort mal référencées, ce qui l'a conduit dans les dépôts de Toulouse et de Bordeaux, mais aussi d'Angleterre et de Hollande -, on connaît mieux aujourd'hui ces « voyageurs qui passent ». Partis d'Espagne, ils gagnent Bordeaux ou Bayonne. Puis, ils s'installent à Toulouse, après 1660. J.B. reconstitue avec précision leurs activités à travers la région, soutenues par le réseau familial qui donne à leurs affaires une dimension internationale. Arrive d'abord le marchand Pierre Souares, négociant en laine venue d'Espagne, qui noue avec les marchands locaux des contacts étroits et fructueux. Puis vient le docteur Balthazar Orobio chassé de la péninsule ibérique par l'Inquisition et trouvant à Toulouse une place à l'université de médecine. Il y fera, avec sa famille, une halte de quelques années avant de trouver un refuge plus définitif à Amsterdam. Là tombent les masques, Balthazar devient Isaac et il pourra se consacrer à son œuvre d'apologiste du judaïsme orthodoxe. D'autres voyageurs trouvent en ce milieu du XVII ${ }^{\mathrm{e}}$ siècle un havre à Toulouse. Mettant au service de leurs affaires leurs liens familiaux, tissant un réseau dense où sentiments, capitaux et marchandises vont et viennent, ils contribuent à revivifier l'activité économique de la capitale du Languedoc. Toulouse, grâce à eux, s'ouvre sur le grand large. Par la Garonne, arrivent des tissus et des soieries qui, via Bordeaux, viennent de Londres ou des ports hollandais. A partir de Toulouse, des myriades de colporteurs acheminent ces biens à travers les campagnes. Les marchands portugais, comme on nommait alors les Marranes, ont su trouver auprès des colporteurs de Saint-Flour des intermédiaires zélés. Roque de Léon semble dominer le groupe. De sa naissance, on ne sait rien de sûr. Né dans une famille habituée à se cacher, il brouille les pistes de sa vie. Il se marie religieusement, dans le rite catholique, à Bordeaux. Il fait partie, selon l'A. de l'aristocratie marrane qui l'aurait envoyé à Toulouse pour ouvrir un commerce.
Autour de lui, gravite tout un groupe - une centaine de personnes. Les trois sœurs Fernandes, Léonore, la femme de Roque, Françoise et Esther se trouvent ainsi le pivot de la communauté, mariées à de riches marchands dont les affaires sont étroitement mêlées. Les familles vivent dans le même quartier, dans le cœur commercial de la ville. Et si les marchands portugais donnent tous les signes d'un catholicisme fervent, choisissant même parfois des parrainages dans la noblesse locale, ils restent attachés au judaïsme, comme le révèle l'enquête de 1685 .

Toutefois, malgré l'audace de ces négociants, le soutien qu'ils trouvent auprès des banques hollandaises, leurs efforts ne sont guère couronnés de succès. Les raisons tiennent autant au contexte économique local qu'au fait qu'ils sont peu intégrés dans le milieu marchand toulousain d'où provient l'attaque en 1685. Ils sont alors dénoncés comme juifs pratiquants. Après avoir tenté de se défendre, ils prennent la fuite sachant très bien que le temps n'est guère propice à la tolérance religieuse : c'est l'année même de la Révocation de l'Édit de Nantes et Toulouse, place forte catholique, n'est guère tendre avec les hérétiques. Bien leur en a pris car le Parlement les condamne au bûcher: dix-huit personnes dont Roque de Léon et sa parentèle auraient dû y trouver place s'ils n'avaient gagné, via Bordeaux, Londres ou Amsterdam. En Hollande, à l'image de Léon, ou en Angleterre, ils renouent ouvertement avec le judaïsme et se lancent à nouveau dans le commerce. C'est ainsi qu'ils participent, de façon non négligeable, à la prospérité des États protestants.

Sur la deuxième hypothèse de départ - la défaite de la France face aux puissances montantes protestantes - on reste un peu plus dubitatif. Car elle est assez peu démontrée par l'ouvrage lui-même. L'analyse détaillée scientifique - des activités des marchands portugais apporte, il est vrai, du grain à la thèse initiale, montrant que la décision politique de proscrire les juifs a mis fin à une expérience économique, annonciatrice d'un capitalisme marchand qui a finalement fait la richesse des États qui ont accueilli les proscrits. Mais on peut se demander si la greffe toulousaine avait quelque chance de prendre, puisque comme le montre l'A., les difficultés n'ont pas tardé à se faire jour, pour des raisons qui tiennent au contexte économique local, bien déprimé après le boom apporté par le pastel au siècle précédent.

L'intérêt essentiel de l'ouvrage est bien l'éclairage qu'il donne sur ces voyageurs qui n'ont fait que passer à Toulouse, leur créativité commerciale s'appuyant sur le fort sentiment 
familial et religieux. C'est à la naissance d'une diaspora que nous assistons, dans l'histoire de laquelle la capitale du Languedoc n'a joué qu'un rôle mineur. Ce travail apporte une belle pierre, précise et érudite, à la construction de l'historiographie des Marranes dans laquelle s'est illustré Gérard Nahon (Métropoles et périphéries séfarades d'Occident: Kairouan, Amsterdam, Bayonne, Bordeaux, Jérusalem, Paris, Cerf, 1993).

Colette Zytnicki.

The Saffron Wave - Democracy and Hindu Nationalism in Modern India. Princeton (N.J.), Princeton University Press, 1999, 293 p. (bibliogr., index, glossaire).

L'ouvrage de T.B.H. porte sur la montée en puissance du mouvement nationaliste hindou de la fin des années 1980 à l'arrivée au pouvoir, en 1998, de la principale formation politique représentant ce courant de la vie politique indienne, le Bharatiya Janata Party. Il a appelé ce moment «la vague safran » parce que cette couleur est celle de l'hindouisme, en tout cas celle qui symbolise cette civilisation aux yeux du mouvement qu'il étudie.

L'intérêt du livre ne vient toutefois pas seulement de la façon dont l'auteur y reconstitue les étapes de l'essor récent du mouvement nationaliste hindou, un processus dont la démolition de la mosquée d'Ayodhya a marqué le point culminant en 1992. En fait, cette chronique politique constitue l'aspect le moins original de The Saffron Wave. Le point fort de l'ouvrage réside d'abord dans le travail de terrain sur lequel repose l'ouvrage. T.B.H. a mené l'enquête dans l'État du Maharashtra, le berceau du mouvement nationaliste hindou. Il y a procédé à d'innombrables interviews, tant en milieu urbain (à Poona notamment), que dans les campagnes.

Le matériau qu'il en a ramené est d'une qualité exceptionnelle et on se prend à regretter qu'il n'y fasse pas davantage référence. En fait, l'A. distille ici l'information à travers des vignettes clairement annoncées par un changement de typographie dans le corps du texte. Ces passages sont à mi-chemin entre l'encadré et le développement intégré au corps du texte. L'ouvrage en compte moins d'une dizaine de deux pages en moyenne. On y voit interagir les différentes branches du mouvement nationaliste hindou au niveau local, on y observe les activités de bienfaisance, leur stratégie d'implantation, leur sociologie élitaire et leur plébéianisation. Non seulement ces morceaux d'anthropologie politique sont trop brefs, mais il manque en outre un examen de la Shiv Sena, un mouvement nationaliste hindou régional au moins aussi bien enraciné que le BJP au Maharashtra.

À partir de cette enquête et de sources écrites, tant primaires que secondaires, l'A. construit une interprétation originale de l'essor du nationalisme hindou. Il y voit d'abord un effet induit de la démocratie indienne et de l'élargissement croissant de sa base sociale : pour lui, la vague safran est une forme de «révolution conservatrice » initiée par des élites de haute caste menacées par la politisation croissante des campagnes et des castes inférieures. Ces élites trouvent dans le nationalisme hindou un contre-feu, des repères sociaux, voire un habillage pour la vieille hiérarchie des castes. En même temps, et c'est là toute l'ambiguïté, le nationalisme hindou est aussi un produit de la démocratisation du système politique indien en tant que mouvement de masse tirant sa force de la rue. Le nationalisme hindou se nourrit ainsi des perversions croissantes de la démocratie indienne, qu'il s'agisse de l'essor du populisme ou de la corruption, véritable faire valoir du BJP - celui-ci aura pu apparaître comme une alternative propre puisqu'extérieure au pouvoir (ou presque) jusqu'aux années 1990.

Parallèlement à ces explications sociales et politiques, T.B.H. ne répugne pas à mobiliser des interprétations relevant de la psychologie collective. Si son usage de Lacan et de son concept de «manque » est peu convaincant, ses références à Zizek éclairent bien la dialectique des relations entre hindous et musulmans. Les nationalistes hindous ont en effet exploité dans les années 1990 des stéréotypes anciens présentant les musulmans comme unis, sûrs d'euxmêmes, virils et dominateurs tandis que les hindous devaient s'organiser pour remédier à leurs divisions et à leurs faiblesses.

Si ce rapport à l'Autre par excellence, le musulman, constitue sans doute la variable clé de l'essor du nationalisme hindou, l'A. aurait cependant dû le resituer dans son contexte : ce discours anti-musulman n'est devenu crédible qu'en raison du renouveau islamiste des années 1980 après la Révolution iranienne et le biais pro-musulman des gouvernements du Congrès qui convoitaient les voix de cette imposante minorité.

Christophe Jaffrelot. 